\title{
myFMH - oder die einfachste Art, seine Dignitätsdaten stets aktuell zur Verfügung zu haben
}

Seit Juni 2006 ist myFMH, das Internetportal für FMH-Mitglieder, in Betrieb. Zeit also, an dieser Stelle die ersten vier Monate Betriebszeit zu analysieren und über den aktuellen Stand zu berichten.

Jacques de Haller, Erika Flückiger

Korrespondenz: Erika Flückiger Leiterin Dienste/FMH Projektgruppe $m y \mathrm{FMH}$ Elfenstrasse 18 CH-3000 Bern 15 erika.flueckiger@fmh.ch

\section{Was ist myFMH?}

Ein Internetportal, geschaffen für FMH-Mitglieder. Mit Ihrem eigenen Schlüssel öffnen Sie das Portal und treten in ein virtuelles Gebäude mit einer Vielzahl von Räumen ein. Vorläufig ist nur der Raum mit dem Namen «Dignitätsdaten» möbliert. Nach und nach werden weitere Räume eingerichtet.

Weshalb haben wir denn ausgerechnet mit den «Dignitätsdaten» begonnen? Einem Thema also, das bei vielen unserer Mitglieder Abwehrreaktionen hervorruft, das man mit «kompliziert» assoziiert, mit «administrativem Leerlauf», «Einschränkung in der ärztlichen Tätigkeit» oder dergleichen? Zugegeben: Solche Reaktionen laden nun wirklich nicht gerade dazu ein, den Schlüssel hervorzunehmen und das Portal zu öffnen. Tatsache ist, dass hier wichtige Fristen zu beachten sind! Deshalb haben wir dieser Applikation oberste Priorität zuordnen müssen.

\section{Deadline 31. Dezember 2006}

Alle Ärztinnen und Ärzte, die seinerzeit bei der Dignitätserhebung Positionen im Besitzstand erfasst haben, müssen bis zum 31. Dezember 2006 in Form einer Selbstdeklaration bestätigen, dass sie die dafür verlangte Fortbildung absolviert haben. Ohne diese Bestätigung werden die Versicherer ab 1. Januar 2007 keine Besitzstandspositionen mehr vergüten.

Die Abwicklung der Dignitätserhebung im Jahr 2003 über den traditionellen Postweg hat grosse Schwierigkeiten bereitet. Deshalb hat sich das Internet als sehr viel effizientere Lösung aufgedrängt; dank seiner Schnelligkeit werden es alle Beteiligten schaffen, die notwendigen Arbeiten fristgerecht zu erledigen. Es versteht sich von selbst, dass wir für den Zugriff auf sensible Daten via Internet die grösstmöglichen Sicherheitsvorkehren getroffen haben.

\section{Wer gibt mir den Schlüssel, damit ich das Portal öffnen kann?}

Damit Sie das Hauptportal öffnen können, um schon mal in das Innere des virtuellen Gebäudes zu gelangen, benötigen Sie Ihren «Identifikationscode» (FMH-ID/FMH-Nummer) und Ihren «Registrierungsschlüssel» (achtstelliger Code, bestehend aus Zahlen und Kleinbuchstaben). Die FMH ist dafür besorgt, dass alle FMH-Mitglieder ihre Registrierungsunterlagen erhalten. In den Monaten Juni und Juli 2006 haben wir - aus Prioritätsgründen, vgl. Deadline weiter oben zunächst einmal allen FMH-Mitgliedern, die effektiv Daten im Besitzstand haben, die Registrierungsunterlagen zugestellt. Alle andern FMH-Mitglieder werden die Registrierungsunterlagen im Laufe des letzten Quartals 2006 erhalten.

\section{Wie kann ich sicher sein, dass kein anderer auf mein Dignitätsprofil zugreift?}

Die Zauberworte heissen «ASAS» und «Sicherheitscodeliste». Mit diesen beiden Sicherheitsvorkehren wird sichergestellt, dass jedes FMHMitglied ausschliesslich auf sein eigenes Dignitätsprofil zugreifen kann.

Wer ein Konto bei Health Info Net hat - das sind aktuell mehr als 10000 FMH-Mitglieder -, profitiert von einem einfacheren und bequemeren Zugang zu seinem Dignitätsprofil. Ist nämlich der ASAS-Client auf dem PC/Notebook aktiviert, wird der Benutzer durch das System automatisch erkannt und eindeutig identifiziert.

Wer noch kein HIN-Konto besitzt, muss nach der Registrierung zunächst ein selbstgewähltes Passwort angeben und sich danach die sogenannte «Sicherheitscodeliste», die angezeigt wird, ausdrucken. Bei jedem künftigen Besuch im myFMH/Dignitätsprofil muss zunächst das Pass- 
Abbildung 1

Sicherheitscodeliste.

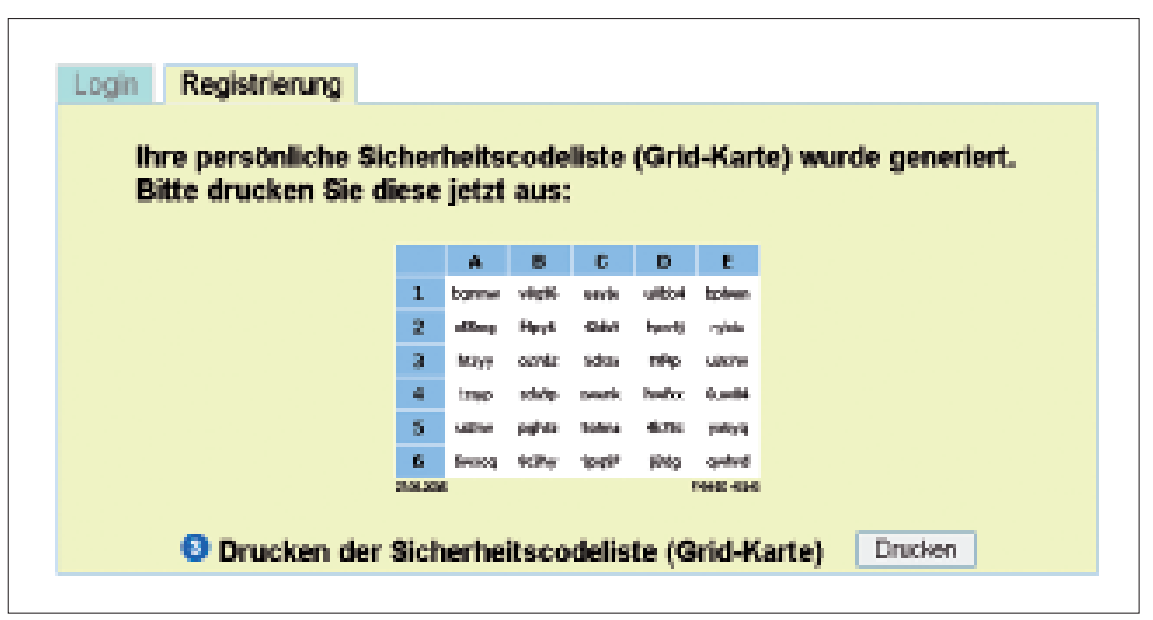

Abbildung 2

Menüpunkte.

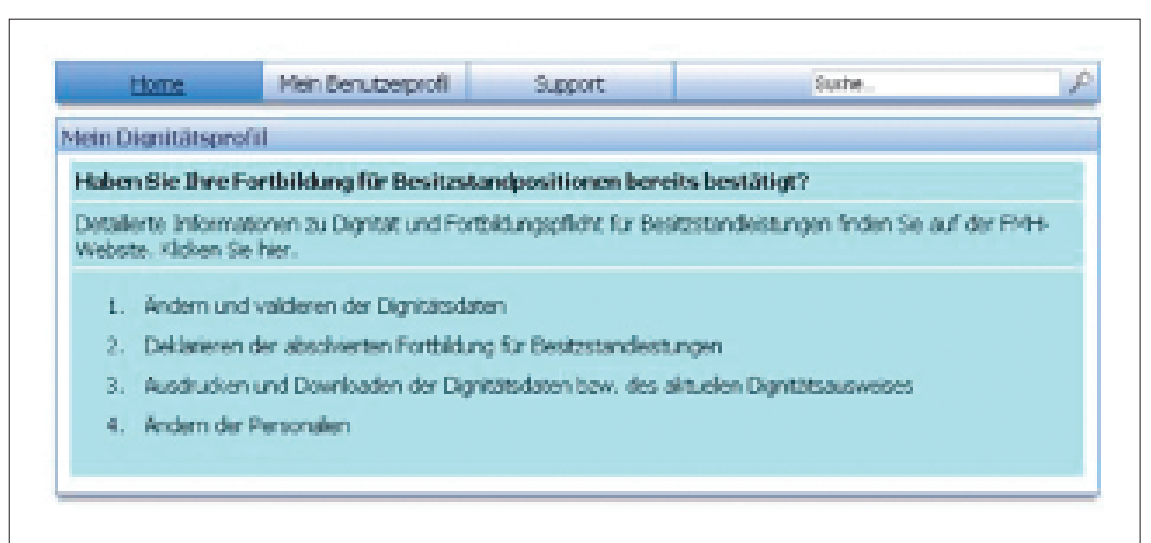

wort wieder eingegeben und danach der vom System verlangte Code aus der Sicherheitscodeliste eingetippt werden - so wie man es sich vom elektronischen Verkehr mit seiner Bank gewohnt ist.

Da mit der Sicherheitscodeliste der Zugriff auf das persönliche Dignitätsprofil von jedem x-beliebigen Computer möglich ist, können sich FMH-Mitglieder mit einem HIN-Account zusätzlich zur Registrierung via ASAS auch noch mit der Sicherheitscodeliste registrieren; damit können sie auf Wunsch auch von einem anderen als ihrem eigenen Computer auf ihr Profil zugreifen.

\section{Was kann oder soll ich mit meinem Dignitätsprofil tun?}

Sie können vier verschiedene Menüpunkte auswählen:

1. Die Dignitätsdaten ändern und validieren Bevor hier die Liste der erfassten Besitzstandspositionen angezeigt wird, werden Sie gebeten, ein paar wenige Fragen zu Ihrer persönlichen Berufsausübung zu beantworten. Diese Angaben sind (mit Ausnahme von zwei Fragen zur ärztlichen Demographie) fakultativ und werden Dritten nicht zugänglich gemacht; aber sie helfen, unsere Datenbasis im Bereich Ärztedemographie zu erweitern und damit die Verhandlungsposition der FMH zu stärken.

Wählen Sie anschliessend aus der Liste der von Ihnen angegebenen Besitzstandleistungen diejenigen aus, die Sie weiterhin abrechnen wollen. Sie verfügen heute über eine mehr als dreijährige Erfahrung mit der Abrechnung nach TARMED. Dies sollte Ihnen die Auswahl derjenigen Positionen erleichtern, die Sie tatsächlich auch weiterhin abrechnen wollen, und für welche Sie die verlangte Fortbildung absolvieren müssen.

\section{Deklarieren der absolvierten Fortbildung für Besitzstandsleistungen}

Das «Dignitätskonzept» (Version 9.0) hält fest, dass für die von der Besitzstandsgarantie abgedeckten Leistungen eine «separate» Fortbildung nachgewiesen werden muss. «Separat» bedeutet, dass diese Fortbildung zusätzlich zu derjenigen zu leisten ist, die für Ihre eigenen Facharzttitel, Schwerpunkte und Fähigkeitsausweise verlangt wird. Damit Sie Ihre Besitzstandspositionen für weitere drei Jahre abrechnen können, muss der Nachweis dieser zusätzlichen Fortbildung bis zum 31. Dezember 2006 erbracht werden. Umfang sowie Art und Weise dieser Fortbildung werden von jedem Arzt und jeder Ärztin in eigener Verantwortung festgelegt. Die Fortbildung muss nicht für jede einzelne Position, sondern kann global für die Gesamtheit der Besitzstandspositionen bestätigt werden [1].

\section{Fortbildung für Abrechnungszertifikate}

Wer sich als Nicht-Facharzttitelträger im Rahmen der Dignitätserhebung einem Facharzttitel zugeordnet hat (Abrechnungszertifikat), muss das Fortbildungsprogramm dieses Titels erfüllen.

Wer die Fortbildungsdeklaration bis zum 31. Dezember 2006 nicht abgegeben hat, muss gewärtigen, dass die entsprechenden Leistungen von den Kostenträgern nicht mehr vergütet werden.

\section{Ausdrucken und Downloaden der Dignitätsdaten bzw. des aktuellen Dignitätsausweises}

Sie können hier Ihr Dignitätsprofil jederzeit einsehen und als aktuellen Dignitätsausweis ausdrucken. Zum Teil gibt es Arbeitgeber (Spitäler), die von ihren angestellten Ärztinnen und Ärzten 
den Dignitätsausweis verlangen. Im myFMH steht Ihnen dieser nun jederzeit aktuell zur Verfügung.

Allerdings mussten wir in letzter Zeit feststellen, dass vermehrt auch Versicherer von den Ärztinnen und Ärzten den Dignitätsausweis verlangen. Wir raten Ihnen dringend davon ab, Ihren Dignitätsausweis an sie weiterzuleiten, auch wenn damit gedroht wird, von Ihnen verrechnete Besitzstandsposition nicht zu vergüten. Die Versicherer haben kein Recht, Ihren Dignitätsausweis zu verlangen! Sie versuchen damit, geltende Verträge zu umgehen. Das vom Bundesrat genehmigte Dignitätskonzept sieht nämlich vor, dass die Versicherer die Besitzstandspositionen in einer Onlineabfrage überprüfen können. Die FMH hat diese Onlineabfragetechnik vertragskonform per Oktober 2004 bereitgestellt. Aus Gründen, die die FMH nicht nachvollziehen kann, wird dieses Onlineinstrument von den Versicherern nicht genutzt, sondern sie versuchen, diese Liste auf Papier von jedem Arzt/jeder Ärztin zu erhalten. Das können wir nicht dulden.

\section{4. Ändern der Personalien}

Hier können Änderungen direkt durch den Arzt/die Ärztin vorgenommen werden. Änderungen in den Personalien werden jedoch - im Gegensatz zu Änderungen im Besitzstand - nicht unmittelbar wirksam. Sie werden zuerst von der Abteilung DLM/Mitgliedschaft überprüft und sind nach etwa zwei Tagen auf dem Personalienblatt im $m y$ FMH sichtbar.

\section{Welche Erkenntnisse haben wir aus den Anfragen an die Helpline gewonnen?}

Obwohl wir $m y$ FMH sowohl intern wie auch in einem Pilotversuch mit 200 FMH-Mitgliedern intensiv getestet haben, sind einige Kinderkrankheiten erst im Massenbetrieb aufgetreten. Meldungen an die Helpline helfen uns, Fehler zu korrigieren und die Benutzerfreundlichkeit zu erhöhen.

Wir sind Ihnen deshalb dankbar, wenn Sie Schwierigkeiten mit der Registrierung haben, diese unserer Helpline über Telefon 0313591259 oder per E-Mail an info-dig@fmh.ch zu melden.

\section{Womit hatten die Benutzer/Benutzerinnen am meisten Schwierigkeiten?}

\section{Registrierung}

Am meisten Anrufe gab es von FMH-Mitgliedern, die ihre Registrierungsunterlagen nicht mehr fanden. Sie haben von uns umgehend neue Unterlagen erhalten. Viele haben, statt sich nur einmalig - nämlich beim ersten Besuch auf der Seite $m y \mathrm{FMH}$ - zu registrieren, den Registrierungsvorgang bei jedem weiteren Besuch wiederholt. Da wir aus Sicherheitsgründen die Benutzung des Registrierungscodes auf 5 Mal beschränkt hatten, war der Zugang beim sechsten Mal gesperrt. Die Betroffenen mussten bei uns neue Registrierungsunterlagen anfordern. Inzwischen haben wir die Benutzung des Registrierungscodes auf max. 10 Mal erhöht.

Viele, die die Registrierung nicht via HIN/ ASAS auswählten, haben es unterlassen, die Sicherheitscodeliste auszudrucken. Beim nächsten Besuch auf myFMH hatten sie den vom System angegebenen Code nicht mehr zur Hand. Vergessen Sie also nicht, die Sicherheitscodeliste unbedingt auszudrucken oder mit der Funktion PrintScreen die Codeliste in ein Worddokument einzufügen und auf dem Computer zu speichern. Sie brauchen sie bei jedem Besuch von myFMH!

\section{Menüführung}

Bei Betriebsbeginn war die Menüführung starr vorgegeben. Bei jedem Besuch musste sich der Benutzer zuerst durch die Personalien «kämpfen». Schwierigkeiten gab es auch bei den obligatorischen Angaben zur Berufstätigkeit und deren Aufteilung nach Stunden und Prozenten.

Die starre Menüführung wurde inzwischen aufgehoben: die Benutzer können jetzt frei wählen, welchen Menüpunkt sie anwählen wollen. Angaben über Änderungen in den Personalien sind nicht mehr obligatorisch. Der stark vereinfachte Block mit den Fragen zur Berufstätigkeit ist nun im Menüpunkt «Ändern und validieren der Dignitätsdaten» integriert und bis auf zwei Demographiefragen ebenfalls nicht mehr obligatorisch auszufüllen.

\section{Speichern von gestrichenen \\ Besitzstandsleistungen}

Einige Benutzer konnten ihre vorgenommenen Streichungen nicht speichern. Erst nach einiger Zeit haben wir herausgefunden, dass ab einer gewissen Anzahl vorgenommener Streichungen das System diese Änderungen nicht übernahm. Das Problem ist inzwischen behoben. Die Betroffenen, bei welchen wir uns auch an dieser Stelle in aller Form entschuldigen, können wir jedoch beruhigen: Die Änderungen, die Sie vorgenommen haben, gingen nicht verloren! Wir haben die Streichungen inzwischen nachgeführt. Die betroffenen FMH-Mitglieder werden wir noch persönlich informieren und bitten, ihr Dignitätsprofil mit den von uns vorgenommenen Änderungen nochmals zu kontrollieren und zu bestätigen. 


\section{Aktueller Stand der Dignitätsprofile in myFMH per Ende September 2006}

Knapp drei Monate vor dem Jahresende ist die Zahl der Ärztinnen und Ärzte, die ihr Dignitätsprofil validiert haben, noch ungenügend. Wir richten deshalb die dringende Bitte an die betroffenen 13000 FMH-Mitglieder, sich so rasch als möglich zu registrieren, danach ihr Dignitätsprofil $\mathrm{zu}$ überarbeiten und $\mathrm{zu}$ validieren und schliesslich die Fortbildung zu bestätigen.

Den wenigen FMH-Mitgliedern, die nicht gewohnt sind, mit Computer zu arbeiten, schicken wir auf Verlangen den Dignitätsausweis zum Überarbeiten, Validieren und zur Bestätigung der Fortbildung auf Papier zu.
Alle anderen können wir nur nochmals auf die Wichtigkeit dieses administrativen Verfahrens hinweisen. Es ist unangenehm, bestimmt, aber absolut notwendig, um weiterhin abrechnen zu können. Besten Dank für Ihre Mitarbeit.

\section{Literatur}

1 Kappeler O, Hänggeli C. Fortbildungen für Besitzstandleistungen. Schweiz Ärztezeitung. 2006; 87(18):768-71.

2 IFAS - Internationale Messe für Arzt- und Spitalbedarf, 24.-27. Oktober 2006, Messe-Zentrum Zürich; Ausschreibungen FMH-Workshops Schweiz Ärztezeitung 2006;87(34; 38; 41;42)

\section{Wir helfen Ihnen!}

Melden Sie sich bei der Helpline, wenn Sie Fragen zur Registrierung haben: Tel. 0313591259 oder Mail an info-dig@fmh.ch

Melden Sie sich bei der Abteilung DLM/Dignität, wenn Sie Fragen zu Ihrem Dignitätsprofil haben: Tel. 0313591111

Registrieren Sie sich in myFMH am FMH-Stand an der IFAS 2006 [2]

\section{4.-27. Oktober 2006 Messe-Zentrum Zürich}

Bringen Sie unbedingt Ihre Registrierungsunterlagen mit! 